EPJ Web of Conferences 43, 04007 (2013)

DOI: $10.1051 /$ epjconf/20134304007

(C) Owned by the authors, published by EDP Sciences, 2013

\title{
The empirical mass distribution of hot B subdwarfs: Implications for stellar evolution theory
}

\author{
V. Van Grootel ${ }^{1,2, a}$, G. Fontaine ${ }^{3}$, S. Charpinet ${ }^{4}$, P. Brassard ${ }^{3}$ and E.M. Green ${ }^{5}$ \\ ${ }^{1}$ Institut d'Astrophysique et de Géophysique, Université de Liège \\ ${ }^{2}$ Chargé de recherches, Fonds de la Recherche Scientifique, FNRS \\ ${ }^{3}$ Département de Physique, Université de Montréal \\ ${ }^{4}$ CNRS, Université de Toulouse, UPS-OMP, IRAP \\ ${ }^{5}$ Steward Observatory, University of Arizona, Tucson
}

\begin{abstract}
Subdwarf B (sdB) stars are hot, compact, and evolved objects that form the very hot end of the horizontal branch, the so-called Extreme Horizontal Branch (EHB). Understanding the formation of sdB stars is one of the remaining challenges of stellar evolution theory. Several scenarios have been proposed to account for the existence of such objects, made of He-burning core surrounded by very thin H-rich envelope. They give quite different theoretical mass distributions for the resulting sdB stars. Detailed asteroseismic analyses, including mass estimates, of 15 pulsating hot B subdwarfs have been published since a decade. The masses have also been reliably determined by light curve modeling and spectroscopy for 7 $\mathrm{sdB}$ components of eclipsing and/or reflection effect binaries. These empirical mass distributions, although based on small-number statistics, can be compared with the expectations of stellar evolution theory. In particular, the two He white dwarfs merger scenario does not seem to be the dominant channel to form isolated sdB stars, while the post-red giant branch scenario is reinforced. This opens new questions on extreme mass loss of red giants to form EHB stars, possibly in connection with the recently discovered close substellar companions and planets orbiting sdB stars.
\end{abstract}

\section{INTRODUCTION}

Subdwarf B (sdB) stars are hot $\left(T_{\text {eff }}=20000-40000 \mathrm{~K}\right)$, compact $(\log g=5.0-6.2)$, and evolved objects that form the very hot end of the horizontal branch, the so-called Extreme Horizontal Branch (EHB). They have He-burning cores surrounded by very thin $(\lesssim 1 \%$ of the total mass) H-rich envelopes. To explain the formation of such objects, it is generally believed that the progenitor loses its envelope almost entirely after the red giant branch (RGB). The residual very thin H-rich envelope has not retained enough mass to sustain shell-burning, and sdB stars evolve directly toward the white dwarf (WD) cooling sequence after He-core exhaustion, without experiencing the Asymptotic Giant Branch (AGB) and Planetary Nebula (PN) phases of usual stellar evolution.

The way a single red giant lose all but a tiny fraction of its envelope close to the tip of the RGB, at the same time as He-burning ignition (via a He-flash or under nondegenerate conditions for progenitors with masses $\gtrsim 2.3 M_{\odot}$ ), is very difficult to explain and requires fine-tuning of the mass loss for unknown or very specific reasons [1] [2]. Because of this, and because of observational evidences showing that a little majority of sdB stars reside in binary systems (e.g., [3] [4]), focus has shifted these last years to binary evolution scenarios, for both the formation of single and binary hot B subdwarfs. These scenarios include evolution through a common envelope (CE), stable Roche lobe overflow (RLOF), and

\footnotetext{
ae-mail: valerie.vangrootel@ulg.ac.be
}

This is an Open Access article distributed under the terms of the Creative Commons Attribution License 2.0, which permits unrestricted use, distribution, and reproduction in any medium, provided the original work is properly cited. 
the merger of two He white dwarfs. In a nutshell, CE evolution would produce sdB stars in close binary systems, while longer period binaries would result from RLOF, and the merger channel would explain the population of single sdB stars. These 3 scenarios have been studied in detail by $[5,6]$ from synthetic binary population simulations that provide, e.g., theoretical mass distributions for the resulting $\mathrm{sdB}$ stars from the various formation channels. The expected mass distributions for CE and RLOF channels, including observational selection effects, peak around $\sim 0.46 M_{\odot}$, while the merger channel leaves a broad mass distribution between $0.4-0.7 M_{\odot}$, with a mean mass of $\sim 0.52 M_{\odot}$. In the weighted mean mass distribution [6], almost all sdBs with masses above $0.5 M_{\odot}$ come from the merger channel.

This "standard model" for the formation of sdB stars successfully explains many observational properties of hot B subdwarfs: the distribution in the orbital period/minimum companion mass diagram, the distributions of orbital period and mass function, the fraction of sdB binaries with WD companions and in globular clusters [7], their birth rate, etc. However, recent observational results challenge this standard binary evolution scenarios, e.g., the discovery of close substellar companions like brown dwarfs around sdB stars [8], or the rotational properties of single sdBs [9]. For a discussion on these challenging observational results, see [10] in these proceedings.

A crucial test for binary evolution scenarios of Han et al. [5, 6] is provided by the comparison with the empirical mass distribution for hot B subdwarfs. Standard measurements of the total mass for $\mathrm{sdB}$ stars are relatively rare, and only possible for $\mathrm{sdB}$ in binaries with favorable conditions such as eclipses or reflection effects. Fortunately, a fraction of sdB stars exhibit nonradial pulsations, offering the opportunity to use asteroseismology in order to determine the mass of sdB stars that are single or that reside in binaries. The first pulsating sdB star, discovered in 1997 [11], shows short-period (1 - 10 min) variations that are typical of sdB pulsators of the first group, the V361 Hya stars. These pulsations are low-order, low-degree acoustic $p$-modes. The pulsating sdBs of the second group have long-period ( $\sim 1-4 \mathrm{~h}$ ) oscillations that correspond to mid/high order, low degree gravity waves ( $g$-modes). The prototype of this class was discovered in 2003 [12], and a dozen is currently being observed from space with the NASA Kepler mission. The first pulsating sdB modeled by asteroseismology was PG 0014+067 in 2001 [13], and since a decade our group has published detailed analyses of 15 pulsating B subdwarfs, including estimates of the masses of these pulsators. Several mass estimates are also available in the literature for sdBs residing in binary systems, from binary light curve modeling combined with accurate spectroscopic measurements. The empirical mass distribution of sdB stars on the basis of this sample is presented in Section 2, as a summary of [14] where all details can be found. Implications for stellar evolution theory on the formation of sdB stars are discussed in Section 3, and finally early conclusions are presented in Section 4.

\section{THE EMPIRICAL MASS DISTRIBUTION OF SdB STARS}

Figure 1 presents the empirical mass distributions of sdB stars, on the basis of the seismic sample (red histogram, 15 pulsators) and the extended sample, including 7 more sdB stars residing in binary systems (blue histogram, 15 pulsators +7 binaries). Let us mention that 4 stars among the 15 pulsators are known to belong to binary systems. The extended sample is therefore made of 11 apparently single sdB stars, and $11 \mathrm{sdBs}$ that are confirmed to reside in binary systems. This binary fraction of $\sim 50 \%$ is similar to the fraction found on large observational surveys (e.g., [3] [4]).

Given that binarity has no incidence on pulsational instabilities [15], and given that our asteroseismic approach has proven to be free of systematic effects for the mass determination [16], there is no a priori reason to suspect that the seismic sample could lead to a biased mass distribution. Indeed this is confirmed a posteriori by Fig. 1 (the blue and red histograms are very similar) and more quantitatively in Table 1, which presents the properties of the empirical mass distributions on the basis of the various subsamples of sdB stars with known masses. All the subsamples (pulsators, sdBs in binaries including pulsators, single sdB stars) have nearly identical mean and median masses, and also similar 1- $\sigma$ ranges where $68.3 \%$ of the stars are found. In particular, no detectable difference is found between the empirical 
Ageing Low Mass Stars: From Red Giants to White Dwarfs

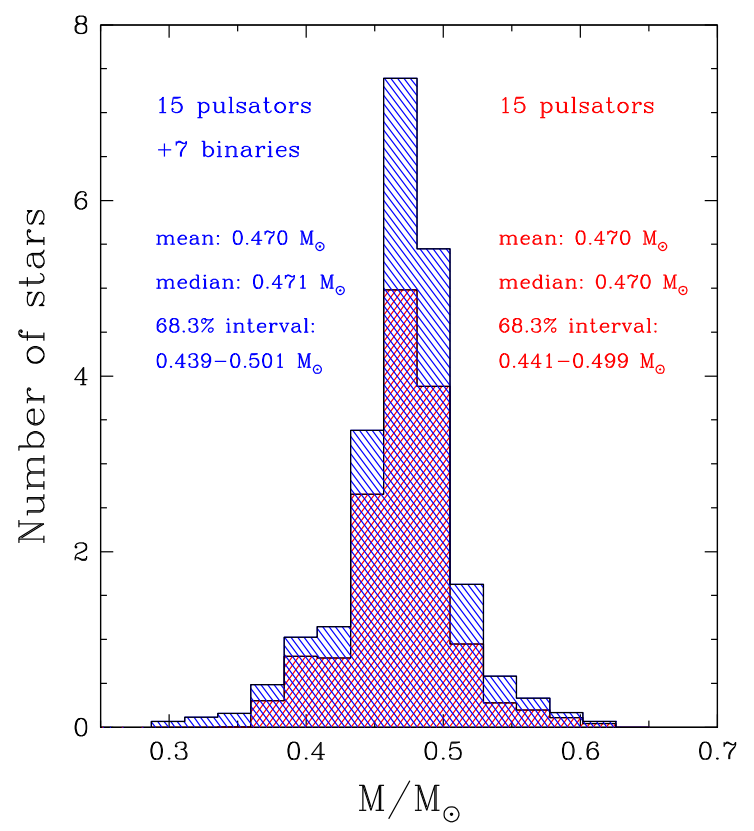

Figure 1. Empirical mass distribution of sdB stars. The red histogram corresponds to the seismic sample of 15 pulsators, while the blue histogram is for the extended sample of $22 \mathrm{sdB}$ stars including the 15 pulsators and $7 \mathrm{sdB}$ stars residing in binaries with masses determined from binary light curve modeling.

Table 1. Properties of the empirical mass distributions of sdB stars for various samples.

\begin{tabular}{lccc}
\hline \hline Sample & $\begin{array}{c}\text { Mean mass } \\
\left(M_{\odot}\right)\end{array}$ & $\begin{array}{c}\text { Median mass } \\
\left(M_{\odot}\right)\end{array}$ & $\begin{array}{c}\text { Range of mass }(68.3 \%) \\
\left(M_{\odot}\right)\end{array}$ \\
\hline Extended (22 stars) & 0.470 & 0.471 & $0.439-0.501$ \\
15 pulsators & 0.470 & 0.470 & $0.441-0.499$ \\
7 binaries (orbits) & 0.468 & 0.474 & $0.431-0.508$ \\
11 binaries (total) & 0.471 & 0.469 & $0.441-0.512$ \\
11 singles & 0.468 & 0.473 & $0.437-0.498$ \\
\hline
\end{tabular}

distributions of single sdB stars (11 stars) and sdBs residing in binaries (11 stars). Details about the various samples and the building of the empirical mass distributions can be found in Sect. 2 and 3 of [14].

\section{IMPLICATIONS FOR STELLAR EVOLUTION THEORY}

\subsection{Comparison with theoretical predictions}

First, a word of caution has to be said about the statistical significance of the current empirical mass distributions. They are based on small numbers of stars (15 in the seismic sample and 22 in the extended, full sample) and should therefore be considered as preliminary. We estimate that the total number of stars involved in each subsample has to reach $\sim 30$ to carry out statistically robust comparisons with expectations from theoretical scenarios for the formation of $\mathrm{sdB}$ stars. However, we find it instructive to already discuss the current results in the framework of stellar evolution theories. 


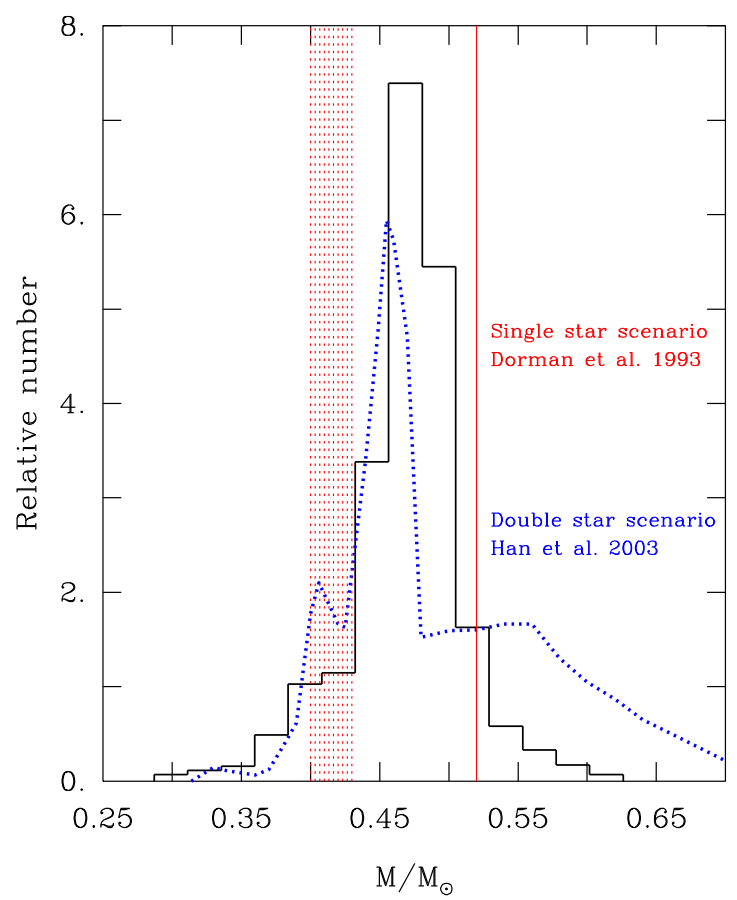

Figure 2. Comparison of the empirical mass distribution of $\mathrm{sdB}$ stars based on the full sample of 22 stars (histogram) with the range of possible masses predicted by single star evolution according to [2]; the red lines mark the (sharp) upper and (fuzzy) lower boundaries of this range. Another comparison is provided by the blue curve which shows one of the predicted mass distributions of sdB stars due to binary evolution according to [6]. The blue curve has been normalized such that the area under it is equal to the area occupied by the histogram (corresponding to the total number of stars in the sample).

Figure 2 compares the empirical mass distribution (histogram) based on the extended sample of 22 stars with the expected range of possible sdB masses from various theories for the formation of such stars. The red boundaries correspond to the calculations for single star evolution, in which a sdB star is formed from a red giant star experiencing a strong mass loss on the RGB [2]. These authors predict a narrow range of possible masses, between around $0.41 M_{\odot}$ and $0.52 M_{\odot}$. The upper limit is rather sharp and corresponds, in the computations of [2], to the mass limit above which a star will ascend the AGB instead of becoming an EHB star. The lower limit is more fuzzy and corresponds to the still uncertain value of the mass required to ignite helium in the stellar core (such a minimum mass depends, e.g., on the metallicity of the progenitor). The blue curve on Fig. 2 is the weighted mass distribution for the 3 binary evolution scenarios of [5,6], including observational selection effects. In this curve (coming from Fig. 22 of [6]), almost all sdBs with masses above $0.5 M_{\odot}$ come from the two He-WD merger channel.

We find that our empirical mass distribution generally agrees quite well with the expectations of stellar evolution theory. The peak of the histogram at $\sim 0.47 M_{\odot}$ is in good agreement with theoretical expectations, both from single star and binary evolution scenarios. The empirical mass distribution is rather symmetric, which can be interpreted as an indication that the merger scenario produces too many massive stars compared to what is observed. This is confirmed by the empirical distribution of the subsample of 11 single stars (see Table 1), which is, with a mean mass of $0.468 M_{\odot}$ and a median mass of $0.473 M_{\odot}$, very different from the expected broad mass distribution $\left(0.4-0.7 M_{\odot}\right.$, with a mean mass around $0.52 M_{\odot}$ ) resulting from the merger scenario [6]. Hence, the merger scenario does not seem to be the dominant channel to form single sdB stars. On the contrary, the empirical mass distributions of 
single stars (11 stars) and sdBs residing in binary systems (11 stars) are very similar (see Table 1). This is an indication that the majority of sdB stars, including the isolated ones, are indeed post-red giant stars. Moreover, the empirical mass distribution strongly peaking at $\sim 0.47 M_{\odot}$ suggests that, in most cases, helium ignition occurs in the stellar core through a He-flash. The peak at $\sim 0.47 M_{\odot}$ also implies that the ZAMS mass of the progenitor is $\lesssim 1.8 M_{\odot}[17][18]^{1}$.

\subsection{Implications for the formation of sdB stars}

If most sdB stars are indeed post-RGB stars that experienced a He-flash, it implies that the core of the red giant progenitor has reached the minimum mass required to trigger it. As a consequence, the loss of the red giant envelope has to occur close to the RGB tip, and not during the RGB phase. Indeed, the He-core has to grow sufficiently through hydrogen shell burning (only possible if the envelope is still present) to reach this minimum mass. In other words, the envelope has to be removed precisely at the same time as the He-flash or later. The chances that synchronization occurs between the two events seem vanishingly small, unless the He-flash contributes to the final kick that would, under certain conditions, expel almost entirely the red giant envelope.

The He-core flash could also happen after some delay, when the star has already left the RGB. Indeed, this is an alternative and somewhat forgotten idea that stars that never quite reach the "standard" He ignition at the RGB tip can achieve a delayed He-core flash while they are collapsing toward the white dwarf stage [1] [19]. Because this delayed He-flash occurs during contraction, at a higher effective temperature than the RGB, it has been called in the literature "hot He-flash", even if the star is still cooler than in its EHB or WD final location. If a sufficient amount of the envelope is removed from a red giant to quench the H-burning shell, the star will begin to collapse, evolving to higher effective temperatures at roughly constant luminosity. If this occurs when the He core is already close to reaching the mass and temperature necessary to ignite helium, then only a small amount of collapse is needed to sufficiently boost the core temperature to cause a He-core flash. These stars are called "early hot flashers" [20], and arrive on the EHB as sdB stars [1]. If the star is located further from the RGB tip when the H-burning shell is stopped, it will need to collapse a lot more to start the He-flash. This mechanism could form hotter stars like He-sdO stars, or even He-core white dwarfs if the central temperature is never high enough to ignite helium.

An observational hint in favor of such a delayed He-flash scenario for sdB stars is related to the HB (Horizontal Branch)/EHB morphology (Fig. 3, from [20]): there is a clear gap in the $\log g-T_{\text {eff }}$ diagram between the hottest Blue HB (BHB) stars and the coolest sdB, EHB stars. This gap suggests a real physical difference between the formation mechanisms of the classic HB stars (that experiences He-flash at the RGB tip) and EHB stars identified as hot B subdwarfs.

In this scenario, the large mass loss for the red giant progenitor to form a sdB star remains to be explained. The presence of a companion in the stellar regime $\left(\gtrsim 0.1 M_{\odot}\right)$ is invoked in binary evolution scenarios, and the envelope of the red giant is removed through a common-envelope phase or through stable Roche lobe overflow [5, 6]. For single stars, such an extreme mass loss along the RGB is more difficult to explain: it may be due to high metallicity environments [1], or to He mixing driven by internal rotation [21]. By increasing the RGB tip luminosity, He mixing leads to enhanced mass loss along the RGB [21]. It has also been proposed that planets and substellar companions like brown dwarfs could be responsible for this enhanced mass loss [22]. The substellar companion deposits angular momentum and energy into the red giant envelope, which is likely to enhance mass loss on the RGB [22]. However, whether those substellar objects are able to eject the envelope is still unclear. In the standard CE-ejection scenario, the amount of energy and angular momentum transferred to the envelope during the CE-phase

\footnotetext{
${ }^{1}$ For progenitor masses $1.8 \lesssim M / M_{\odot} \lesssim 2.3$, the He-core burning mass, still experiencing a He-flash, decreases to as low as $\sim 0.3$ $M_{\odot}$; while for ZAMS masses above $\sim 2.3 M_{\odot}$, He ignites under nondegenerate conditions, and the He-core mass increases with the mass of the progenitor [17] [18].
} 


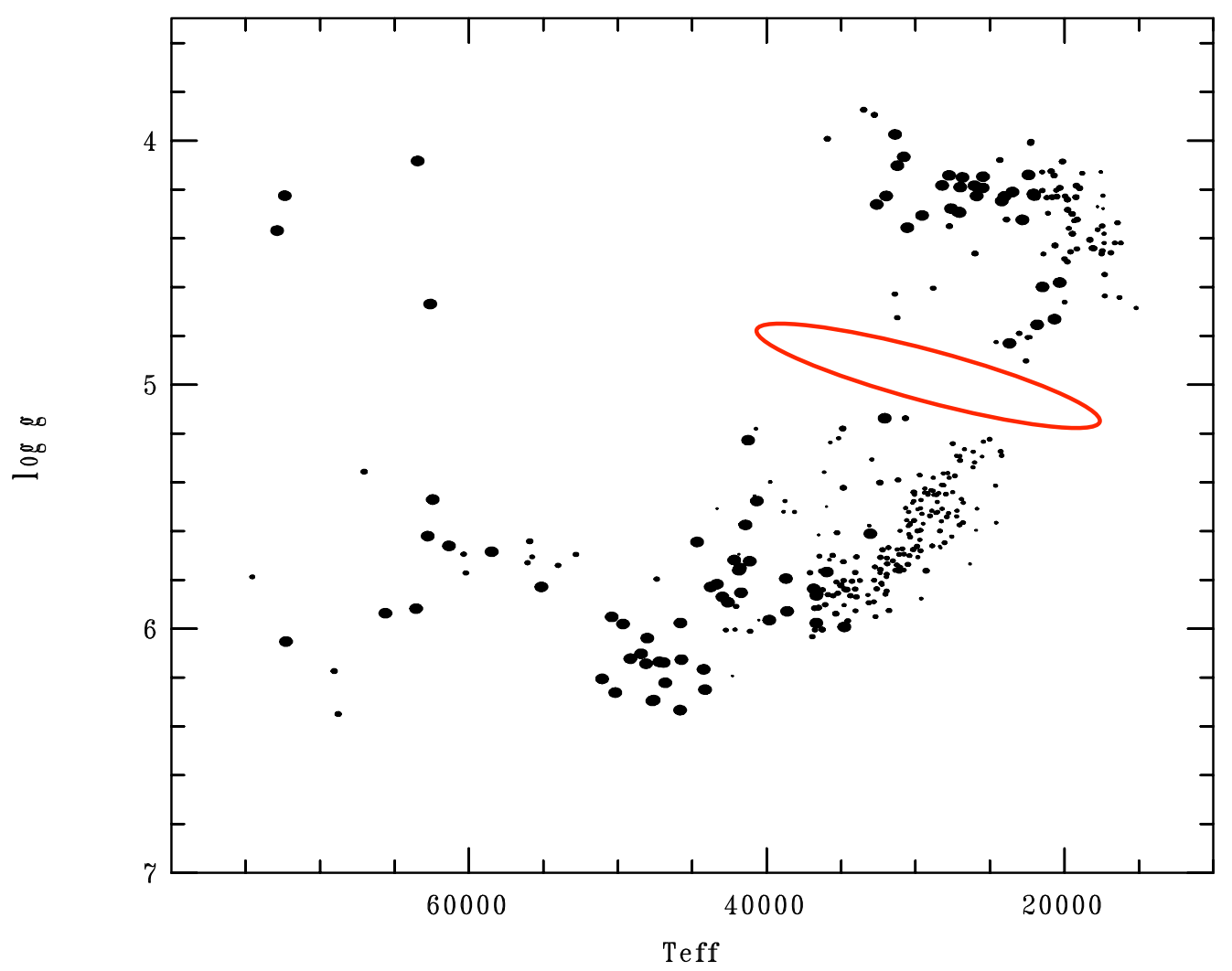

Figure 3. $\log g-T_{\text {eff }}$ diagram for all non-composite spectra in a low resolution, homogeneous survey of hot subdwarf candidates. The sizes of the plotted points are proportional to the derived atmospheric He abundances. The red circle shows the clear gap between the hottest BHB stars and the coolest EHB, sdB stars. Figure from [20].

scales with the mass of the engulfed companion [5]. [23] have recently suggested that a massive enough substellar companion is able to eject the CE if the energy description of CE ejection (i.e. the alphadescription) still works, and the sdB progenitor mass is in the range 0.8-1.26 $M_{\odot}$ [23].

These last years, observational evidences for the presence of substellar companions for sdB stars have been found. Among other candidates, two sdB+brown dwarf short-period binaries have been confirmed in the course of the MUCHFUSS project [8]. Furthermore, [24] have recently determined that the number fraction of substellar companions for $\mathrm{sdB}$ stars seems to be comparable to the one of low-mass main sequence stars. Two very close $(<0.008$ UA) Earth-sized planets have been found orbiting the pulsating sdB star KPD 1943+4058 observed by Kepler [25]. These bodies are believed to have survived deep immersion in the red giant envelope. They may be the remains of one or two evaporated or disrupted giant planets that were transported closer to the star during the engulfment, and that triggered the mass loss necessary for the formation of the sdB star [25] [26] [27] [28].

It is estimated that $\sim 7 \%$ of main sequence stars have close-in giant planets [29], and at least $50 \%$ have a super-Earth or a Neptune $\left(\gtrsim 10 M_{\oplus}\right)$ with periods shorter than 50 days [30], that will be engulfed during the red giant phase: such configurations where a sdB is formed from star/planet(s) interaction(s) may then be fairly common. The companions do not necessarily have to survive the CE phase, leading to "real" single sdB stars, or sdB stars with substellar companions that are usually very difficult to detect. We suggest here that the substellar channel for CE evolution could form a significant fraction of sdB stars. 
Ageing Low Mass Stars: From Red Giants to White Dwarfs

\section{SUMMARY AND CONCLUSIONS}

The empirical mass distribution of sdB stars, although based on small-number statistics (15 pulsators in the seismic sample, 22 stars in the extended sample), is strongly peaked at $\sim 0.47 M_{\odot}$. This is in good agreement with theoretical expectations, both from single star and binary evolution. The distribution is rather symmetric, indicating that the He-WD merger scenario predicts too many massive $\left(\gtrsim 0.5 M_{\odot}\right)$ $\mathrm{sdB}$ stars compared to what is observed. This is confirmed by the mass distribution of the 11 single stars in our sample, which is also strongly peaked at $\sim 0.47 M_{\odot}$ and is very different from the broad $\left(0.4-0.7 M_{\odot}\right)$ theoretical mass distribution for the He-WD merger scenario. Hence, the latter does not seem to be the dominant way to form single sdB stars. The same conclusion has recently been reached by [9], who measured the projected rotational velocities of a large sample of single and wide binary sdBs. All these stars turned out to be slow rotators $\left(v_{r o t} \sin i<10 \mathrm{~km} \mathrm{~s}^{-1}\right)$, which is difficult to understand in the context of the merger scenario, because merger remnants are likely to spin fast.

The fact that the empirical mass distribution strongly peaks at $\sim 0.47 M_{\odot}$ suggests that the majority of sdB stars should be post-RGB stars that have experienced a He-flash, and implies that the ZAMS mass of the progenitor is below $\sim 1.8 M_{\odot}$. The He-core flash could happen after the progenitor leaves the RGB, as "hot flashers" during the star is collapsing, instead of a classic flash at the RGB tip. Or, in a scenario where both events are synchronized, the flash itself could in fact contribute to expel the envelope of the red giant star, provided the latter is in a configuration where its binding has been weakened.

No detectable difference is found between the empirical distributions of single sdB stars (11 stars) and of sdBs residing in binaries (11 stars). This $50 \%$ binary fraction is representative of what is found in $\mathrm{sdB}$ stars in general. This suggests that the formation of (apparently) single sdB stars is similar to the one of sdBs in binary systems. Indeed, substellar companions like brown dwarfs, giant planets or remnant planetary cores have been found orbiting sdB stars these last years. Such companions, when engulfed in the red giant envelope and migrating inward, deposit orbital energy (and angular momentum) into the envelope and could contribute to unbind it and possibly form a sdB star. As a bonus, the ejection of the envelope takes away some angular momentum of the system (which mainly resides in orbiting objects), which may explain why single and wide binary sdB stars are all slow rotators.

The empirical mass distribution for $\mathrm{sdB}$ stars, from which many fundamental questions can be investigated as illustrated here, needs to be more statistically robust. This is one of our long-term goals, in particular by pursuing the investigation of these objects with asteroseismology. Additional targets (already observed from the ground and currently monitored from space by Kepler) are waiting for detailed seismic analyses. This is the necessary step to confirm/infirm the first tendencies presented here.

\section{References}

[1] D’Cruz, N.L., Dorman, B., Rood, R.T., \& O’Connell, R.W., ApJ 466, (1996) 359

[2] Dorman, B., Rood, R.T., \& O’Connell, R.W., ApJ 419, (1993) 596

[3] Allard, F., Wesemael, F., Fontaine, G., et al., AJ 107, (1994) 1565

[4] Maxted, P., Heber, U., Marsh, T., \& North, R., MNRAS 326, (2001) 1391

[5] Han, Z., Podsiadlowski, P., Maxted, P.F.L., Marsh, T.R., \& Ivanova, N., MNRAS 336, (2002) 449

[6] Han, Z., Podsiadlowski, P., Maxted, P.F.L., \& Marsh, T.R., MNRAS 341, (2003) 669

[7] Han, Z.A\&A 484, (2008) L31

[8] Geier, S., Schaffenroth, V., Drechsel, H., et al., ApJL 731, (2011) 22

[9] Geier, S., \& Heber, U., A\&A 149, (2012) 543

[10] Geier, S., EPJ Web of Conferences, these proceedings

[11] Kilkenny, D., Koen, C., O’Donoghue, D., \& Stobie, R. S., MNRAS 285, (1997) 640

[12] Green, E.M., Fontaine, G., Reed, M.D., et al. ApJL, 583, (2003) 31

[13] Brassard, P., Fontaine, G., Billéres, M., et al., ApJ 563, (2001) 1013 
[14] Fontaine, G., Brassard, P., Charpinet, S., et al., A\&A 539, (2012) 12

[15] Billères, M., Fontaine, G., Brassard, P., et al., ApJL 487, (1997) 81

[16] Charpinet, S., Van Grootel, V., Reese, D., et al., A\&A 489, (2008) 377

[17] Sweigart, A., Greggio, L., \& Renzini, A., ApJ 364, (1990) 527

[18] Castellani, V., Degl'Innocenti, S., Girardi, L., et al., A\&A 354, (2000) 150

[19] Castellani, M., \& Castellani, V., ApJ 407, (1993) 649

[20] Green, E.M., Fontaine, G., Hyde, E.A., et al., ASP Conf. Ser. 392, (2008) 75

[21] Sweigart, A., ApJL 474, (1997) 23

[22] Soker, N., AJ 116, (1998) 1308

[23] Han, Z., Chen, X., Lei, Z., \& Podsiadlowski, P., ASP Conf. Ser. 452, (2012) 3

[24] Geier, S., Classen, L., Brünner, P., et al., ASP Conf. Ser. 452, (2012) 153

[25] Charpinet, S., Fontaine, G., Brassard, P., et al., Nature 480, (2011) 496

[26] Bear, E., \& Soker, N., ApJL 749, (2012) 14

[27] Kempton, E., Nature 480, (2011) 460

[28] Passy, J.-L., Mac Low, M.-M., \& De Marco, O., ApJL 759, (2012) 30

[29] Udry, S., \& Santos, N., ARA\&A 45, (2007) 397

[30] Figueira, P., Marmier, M., Boué, G., et al., A\&A 541, (2012) 139 